\title{
DARBOUX TRANSFORMATIONS AND ISOMETRIC IMMERSIONS OF RIEMANNIAN PRODUCTS OF SPACE FORMS
}

\author{
Qun He and Yi-Bing Shen
}

\begin{abstract}
By using the Darboux transformation in Soliton theory, we give the explicit construction for local isometric immersions of the Riemannian product $M_{1}^{n_{1}}\left(c_{1}\right) \times M_{2}^{n_{2}}\left(c_{2}\right)$ into space forms $M^{m}(c)$ with flat normal bundle via purely algebraic algorithm.
\end{abstract}

\section{§0. Introduction}

The problem on isometric immersions of Riemannian manifolds into space forms is an interesting classical problem. There are a lot of nonexistence results in this area $([\mathrm{CK}],[\mathrm{Hi}],[\mathrm{Pe}],[\mathrm{Xa}]$, etc.). Recently, it has been found that the integrability condition for isometric immersions of space forms, i.e., GaussCodazzi-Ricci equations, is equivalent to the condition of a family of connections to be flat ([FP], [Ter]). This enable us to apply the soliton theory to the study of some problems on isometric immersions of space forms. For instance, some Bäcklund transformations for such isometric immersions were considered in [FP] and $[\mathrm{TU}]$. The Darboux transformation method for the explicit expressions of such isometric immersions via purely algebraic algorithm has been given in $[\mathrm{Zh}$, HS], respectively. It is natural to consider the problem on isometric immersions of Riemannian product $M_{1}^{n_{1}}\left(c_{1}\right) \times M_{2}^{n_{2}}\left(c_{2}\right)$ into space forms $M^{m}(c)$.

The purpose of this paper is to apply the Darboux transformation method to the study of local isometric immersions from the Riemannian product of space forms into space forms with flat normal bundle. Some fundamental theory on local isometric immersions of $M_{1}^{n_{1}}\left(c_{1}\right) \times M_{2}^{n_{2}}\left(c_{2}\right)$ into $M^{m}(c)$ with $c=$ $c_{1} c_{2} /\left(c_{1}+c_{2}\right)$ is developed in $\S 1$. In $\S 2$, a zero curvature condition for such local isometric immersions is given, i.e., a family of connection 1-forms including one parameter are flat. It is different from $[\mathrm{FP}]$. In $\S 3$, the Darboux transformations for the explicit expressions of such isometric immersions are shown. This is

1991 Mathematics Subject Classification: Primary 53C42; Secondary 35Q58.

Keywords and phrases: Riemannian product, isometric immersion, Darboux transformation.

Project supported by the National Natural Science Foundation of China (10271106), the Scientific Foundation of the National Education Department of China (1999033554), and the Natural Science Foundation of Zhejiang Province.

Received February 25, 2002. 
a purely algebraic algorithm. Finally, in $\S 4$, we give an explicit construction of such isometric immersions from a trivial (degenerated) isometric immersion via the Darboux transformation for the twisted $s o(p, q, r)$-hierarchy. It is possible that the method of this paper may be used to study local isometric immersions of the Riemannian product of several space forms into space forms.

\section{§1. Isometric immersions of Riemannian products}

Let $M^{n}(c)$ denote an $n$-dimensional space form of constant curvature $c$. Let $\mathbf{r}_{c}: M^{m}(c) \rightarrow \boldsymbol{R}_{c}^{m+1}$ be the following standard isometric embedding:

$$
\begin{aligned}
& M^{m}(c)=\left\{\left(x_{0}, x_{1}, \ldots, x_{m}\right) \in \boldsymbol{R}^{m+1} \mid \sum_{A=1}^{m} x_{A}^{2}+x_{0}^{2}=\frac{1}{c^{2}}\right\} \text { for } c>0, \\
& M^{m}(c)=\left\{\left(x_{0}, x_{1}, \ldots, x_{m}\right) \in \boldsymbol{R}^{m, 1} \mid \sum_{A=1}^{m} x_{A}^{2}-x_{0}^{2}=-\frac{1}{c^{2}}\right\} \quad \text { for } c<0, \\
& M^{m}(0)=\left\{\left(x_{0}, x_{1}, \ldots, x_{m}\right) \in \boldsymbol{R}^{m+1} \mid x_{0}=0\right\} .
\end{aligned}
$$

Consider a locally isometric immersion $\varphi: M_{1}^{n_{1}}\left(c_{1}\right) \times M_{2}^{n_{2}}\left(c_{2}\right) \supset U \rightarrow M^{m}(c)$ with $c_{2} \neq 0$ and $c=c_{1} c_{2} /\left(c_{1}+c_{2}\right)$, where $m>n_{1}+n_{2}=n$ and, without loss of generality, $c= \pm 1$ or 0 . We shall make use of the following convention on the ranges of indices unless otherwise stated:

$$
\begin{gathered}
i, j, k, \ldots=1, \ldots, n_{1} ; \quad q, s, t, \ldots=n_{1}+1, \ldots, n ; \\
I, J, K, \ldots=1, \ldots, n ; \quad r=1,2 ; \quad \alpha, \beta, \ldots=1, \ldots, m-n .
\end{gathered}
$$

Then the composition map $\mathbf{r}=\mathbf{r}_{c} \circ \varphi: U \rightarrow \boldsymbol{R}_{c}^{m+1}$ is a local isometric immersion into $\boldsymbol{R}_{c}^{m+1}$. Set

$$
J_{c}=\left(\begin{array}{cc}
c & 0 \\
0 & I_{m}
\end{array}\right), \quad s o_{c}(m+1)=\left\{X \in \operatorname{sl}(m+1, \boldsymbol{R}) \mid X J_{c}+J_{c} X^{T}=0\right\} .
$$

Denote by $S O_{c}(m+1)$ the Lie group of which the Lie algebra is $s o_{c}(m+1)$. Consider a framing field $\Psi=\left(e_{0}, e_{1}, \ldots, e_{m}\right): U \rightarrow S O_{c}(m+1)$ in $\boldsymbol{R}_{c}^{m+1}$ so that $\mathbf{r}=J_{c}^{2} e_{0},\left\{e_{i}\right\}$ and $\left\{e_{s}\right\}$ are tangent to $M_{1}^{n_{1}}\left(c_{1}\right)$ and $M_{2}^{n_{2}}\left(c_{2}\right)$ respectively, and $\left\{e_{n+\alpha}\right\}$ are normal to $M_{1} \times M_{2}$ in $M^{m}(c)$. Clearly, $e_{0}$ is normal to $M^{m}(c)$ for $c \neq 0$. Let $\Xi=\Psi^{-1} d \Psi$ be the pull back of the Maurer-Cartan form of $S O_{c}(m+1)$ by $\Psi$, which is an $s o_{c}(m+1)$-valued 1 -form. We then have

$$
\left\{\begin{array}{l}
d \Psi=\Psi \Xi, \\
\Psi(0)=I_{m+1},
\end{array} \quad \text { where } \Xi=\left(\begin{array}{cccc}
0 & -c \theta_{1}^{T} & -c \theta_{2}^{T} & 0 \\
\theta_{1} & \omega_{1} & 0 & \beta_{1} \\
\theta_{2} & 0 & \omega_{2} & \beta_{2} \\
0 & -\beta_{1}^{T} & -\beta_{2}^{T} & \eta
\end{array}\right),\right.
$$

where $\theta_{1}=\left(\theta^{1}, \ldots, \theta^{n_{1}}\right)^{T}$ and $\theta_{2}=\left(\theta^{n_{1}+1}, \ldots, \theta^{n}\right)^{T}$ are dual fields of $\left\{e_{i}\right\}$ and $\left\{e_{s}\right\}$ respectively, $\omega_{1}=\left(\omega_{i j}\right)$ and $\omega_{2}=\left(\omega_{s t}\right)$ are the Levi-Civita connection 1-forms of 
$M_{1}^{n_{1}}\left(c_{1}\right)$ and $M_{2}^{n_{2}}\left(c_{2}\right)$ respectively, $\beta_{1}=\left(\omega_{i, n+\alpha}\right)$ and $\beta_{2}=\left(\omega_{s, n+\alpha}\right)$ are the second fundamental form of the isometric immersion $\varphi, \eta=\left(\omega_{n+\alpha, n+\beta}\right)$ is the normal connection of $\varphi$.

The integrability condition for the existence of such a framing field $\Psi$ is that $\Xi$ satisfies the Maurer-Cartan equation

$$
d \Xi+\Xi \wedge \Xi=0
$$

i.e.,

$$
\begin{aligned}
& d \theta_{r}+\omega_{r} \wedge \theta_{r}=0, \\
& d \omega_{r}+\omega_{r} \wedge \omega_{r}-c \theta_{r} \wedge \theta_{r}^{T}-\beta_{r} \wedge \beta_{r}^{T}=0, \\
& d \beta_{r}+\beta_{r} \wedge \eta+\omega_{r} \wedge \beta_{r}=0, \\
& d \eta+\eta \wedge \eta-\beta_{1}^{T} \wedge \beta_{1}-\beta_{2}^{T} \wedge \beta_{2}=0, \\
& \beta_{1} \wedge \beta_{2}^{T}+c \theta_{1} \wedge \theta_{2}^{T}=0, \\
& \theta_{1}^{T} \wedge \beta_{1}+\theta_{2}^{T} \wedge \beta_{2}=0 .
\end{aligned}
$$

Since $M_{1}^{n_{1}}\left(c_{1}\right)$ and $M_{2}^{n_{2}}\left(c_{2}\right)$ have constant curvatures $c_{1}$ and $c_{2}$ respectively, then

$$
d \omega_{r}+\omega_{r} \wedge \omega_{r}=c_{r} \theta_{r} \wedge \theta_{r}^{T} .
$$

It follows from $(1.2)_{2}$ and $(1.3)$ that

$$
\beta_{r} \wedge \beta_{r}^{T}+\left(c-c_{r}\right) \theta_{r} \wedge \theta_{r}^{T}=0 .
$$

If the normal bundle of $\varphi$ is flat, then

$$
d \eta+\eta \wedge \eta=\beta_{1}^{T} \wedge \beta_{1}+\beta_{2}^{T} \wedge \beta_{2}=0 .
$$

Set

$$
\begin{gathered}
\varepsilon_{r}=\operatorname{sgn}\left(c_{r}\right), \quad \kappa_{r}= \begin{cases}1 & \text { for } c_{r}=0, \\
\sqrt{\left|c_{r}\right|} & \text { for } c_{r} \neq 0,\end{cases} \\
\varepsilon=\operatorname{sgn}\left(c-c_{2}\right), \quad v_{1}=\varepsilon c \sqrt{\left|c-c_{1}\right|}, \quad v_{2}=\sqrt{\left|c-c_{2}\right|} .
\end{gathered}
$$

Clearly, we see that $\varepsilon_{2}, v_{2}, \varepsilon, \kappa_{r} \neq 0$, and $\varepsilon=\operatorname{sgn}\left(c-c_{1}\right)=\operatorname{sgn}\left(c-c_{2}\right)$ when $c \neq 0$. Noting that $c^{2}=1$ or 0 , thus, $(1.2)_{5}$ and (1.4) can be rewritten as

$$
\begin{aligned}
& \beta_{1} \wedge \beta_{2}^{T}+\varepsilon v_{1} v_{2} \theta_{1} \wedge \theta_{2}^{T}=0, \\
& \beta_{r} \wedge \beta_{r}^{T}+\varepsilon v_{r}^{2} \theta_{r} \wedge \theta_{r}^{T}=0 .
\end{aligned}
$$

Definition 1.1. Let $\varphi: M_{1}^{n_{1}} \times M_{2}^{n_{2}} \rightarrow M^{m}$ be an isometric immersion. If Weingarten endomorphisms for $\varphi$ preserve $T M_{1}$ and $T M_{2}$ invariant, respectively, i.e., $\beta_{1}$ and $\beta_{2}$ can be expressed linearly by $\theta_{1}$ and $\theta_{2}$ respectively, then the second fundamental form of $\varphi$ is called to be separable.

For $c \neq 0$ and $\varepsilon>0,(1.7)$ implies that the second fundamental form $\psi_{\alpha}=$ $\sum_{I} \omega_{I, n+\alpha} \otimes \theta^{I}$ and the symmetric bilinear form $\psi=v_{1} \theta_{1}^{T} \otimes \theta_{1}+v_{2} \theta_{2}^{T} \otimes \theta_{2}$ are 
exteriorly orthogonal, and they can be simultaneously diagonalized for $m=$ $2 n-1$ by virtue of Cartan's theorem [Mo]. Thus, the immersion $\varphi$ has flat normal bundle. Hence, in the way similar to the case of isometric immersions of space forms $[\mathrm{Mo}]$, we have the following

Proposition 1.2. There is no isometric immersion $\varphi: M_{1}^{n_{1}}\left(c_{1}\right) \times M_{2}^{n_{2}}\left(c_{2}\right) \rightarrow$ $M^{2 n-2}(c)$ with $c=c_{1} c_{2} /\left(c_{1}+c_{2}\right)>c_{1}$. Moreover, if $\varphi: M_{1}^{n_{1}}\left(c_{1}\right) \times M_{2}^{n_{2}}\left(c_{2}\right) \rightarrow$ $M^{2 n-1}(c)$ is a local isometric immersion with $c=c_{1} c_{2} /\left(c_{1}+c_{2}\right)>c_{1}$, then the normal connection of $\varphi$ is flat.

In general, if the isometric immersion $\varphi$ has flat normal bundle, then the second fundamental of $\varphi$ can be simultaneously diagonalized. In addition, if the second fundamental form of $\varphi$ is separable, then we can choose the tangent frame fields $\left\{e_{i}\right\}$ to $M_{1}^{n_{1}}\left(c_{1}\right)$ and $\left\{e_{s}\right\}$ to $M_{2}^{n_{2}}\left(c_{2}\right)$ such that $\omega_{i, n+\alpha}=b_{i \alpha} \theta^{i}$ and $\omega_{s, n+\alpha}=b_{s \alpha} \theta^{s}$. Moreover, we can choose a parallel normal frame fields $\left\{e_{\alpha}\right\}$ so that $\eta=0$.

On putting

$$
\omega_{i j}=\sum_{k} \Gamma_{i j}^{k} \theta^{k}, \quad \omega_{s t}=\sum_{q} \Gamma_{s t}^{q} \theta^{q},
$$

we have from (1.2), (1.4) and (1.7)

$$
\begin{aligned}
& \sum_{\alpha} b_{i \alpha} b_{j \alpha}+\varepsilon v_{1}^{2}=0, \quad(i \neq j) \\
& \sum_{\alpha} b_{s \alpha} b_{t \alpha}+\varepsilon v_{2}^{2}=0, \quad(s \neq t) \\
& \sum_{\alpha} b_{i \alpha} b_{s \alpha}+\varepsilon v_{1} v_{2}=0, \\
& \left(b_{i \alpha}-b_{j \alpha}\right) \Gamma_{i j}^{k}=\left(b_{i \alpha}-b_{k \alpha}\right) \Gamma_{i k}^{j}, \quad(i, j, k \neq) \\
& \left(b_{s \alpha}-b_{t \alpha}\right) \Gamma_{s t}^{q}=\left(b_{s \alpha}-b_{q \alpha}\right) \Gamma_{s q}^{t}, \quad(q, s, t \neq) \\
& e_{j}\left(b_{i \alpha}\right)=\left(b_{j \alpha}-b_{i \alpha}\right) \Gamma_{i j}^{i}, \quad(i \neq j) \\
& e_{t}\left(b_{s \alpha}\right)=\left(b_{t \alpha}-b_{s \alpha}\right) \Gamma_{s t}^{s}, \quad(s \neq t) \\
& e_{s}\left(b_{i \alpha}\right)=e_{i}\left(b_{s \alpha}\right)=0 .
\end{aligned}
$$

Set

$$
\begin{aligned}
& B_{1}=\left(b_{i \alpha}\right), \quad B_{2}=\left(b_{s \alpha}\right), \quad V_{1}=(\underbrace{v_{1}, \ldots, v_{1}}_{n_{1}})^{T}, \quad V_{2}=(\underbrace{v_{2}, \ldots, v_{2}}_{n_{2}})^{T}, \\
& B=\left(\begin{array}{ll}
B_{1} & V_{1} \\
B_{2} & V_{2}
\end{array}\right), \quad \tilde{J}_{\varepsilon}=\left(\begin{array}{cc}
I_{m-n} & 0 \\
0 & \varepsilon
\end{array}\right) .
\end{aligned}
$$


Then we see from $(1.8)_{1-3}$ that $B \tilde{J} B^{T}=\operatorname{diag}\left(\rho_{1}, \ldots, \rho_{n}\right)$, where

$$
\rho_{i}=\sum_{\alpha} b_{i \alpha}^{2}+\varepsilon v_{1}^{2}, \quad \rho_{s}=\sum_{\alpha} b_{s \alpha}^{2}+\varepsilon v_{2}^{2} .
$$

Lemma 1.3. Let $\varphi: M_{1}^{n_{1}}\left(c_{1}\right) \times M_{2}^{n_{2}}\left(c_{2}\right) \supset U \rightarrow M^{m}(c)$ be a locally isometric product immersion with flat normal bundle and the separable second fundamental form. Assume that $\rho_{I} \neq 0$ for all I where $\rho_{I}$ are smooth functions defined by (1.10). Then there exist a line of curvature coordinates $\left(x_{i}, x_{s}\right)$ on $U$ such that the first and second fundamental forms of $\varphi$ can be given by

$$
\begin{aligned}
& I=\sum_{i} a_{i}^{2} d x_{i}^{2}+\sum_{s} a_{s}^{2} d x_{s}^{2}, \\
& I I=\sum_{\alpha}\left(\sum_{i} a_{i}^{2} b_{i \alpha} d x_{i}^{2}+\sum a_{s}^{2} b_{s \alpha} d x_{s}^{2}\right) e_{n+\alpha} .
\end{aligned}
$$

Proof. Since $\rho_{I} \neq 0$ then we can write $\rho_{I}= \pm\left(a_{I}\right)^{-2}$ with $a_{I}>0$. It follows from (1.8) and (1.10)

$$
e_{j}\left(a_{i}\right)=a_{i} \Gamma_{i j}^{i}, \quad e_{t}\left(a_{s}\right)=a_{s} \Gamma_{s t}^{s} .
$$

For any point $x \in U$, if we choose $e_{n+1}$ at $x$ so that $b_{11}(x) \neq 0, b_{12}(x)=\cdots=$ $b_{1, m-n}(x)=0$, then it follows from $(1.8)_{1}$ and $(1.10)_{1}$ that

$$
b_{i 1}(x)=-\frac{\varepsilon v_{1}^{2}}{b_{11}(x)} \quad(i \neq 1), \quad b_{11}^{2}(x)+\varepsilon v_{1}^{2}=\rho_{1} \neq 0 .
$$

By taking $\alpha=j=1$ in $(1.8)_{4}$, we see from (1.13) that $\Gamma_{i 1}^{k}(x)=0$. Since $x$ is arbitrary, then it follows that $\Gamma_{i 1}^{k}=0$ for $i, k, 1$ distinct. We know that the components $\Gamma_{i j}^{k}$ of $\omega_{i j}$ are independent of the choice of the fields of normal frames. Thus, we have $\Gamma_{i j}^{k}=0$ for $i, j, k$ distinct. By the same reason, we have also $\Gamma_{s t}^{q}=0$ for $q, s, t$ distinct. Hence, by using (1.12) and the skew-symmetry, we conclude that

$$
\omega_{i j}=\frac{e_{j}\left(a_{i}\right)}{a_{i}} \theta^{i}-\frac{e_{i}\left(a_{j}\right)}{a_{j}} \theta^{j}, \quad \omega_{s t}=\frac{e_{t}\left(a_{s}\right)}{a_{s}} \theta^{s}-\frac{e_{s}\left(a_{t}\right)}{a_{t}} \theta^{t} .
$$

As the same as in [Mo], it is easy from (1.14) to see that there exist a line of curvature coordinates $\left(x_{i}, x_{s}\right)$ on $U$ so that $\partial / \partial x_{i}=a_{i} e_{i}, \partial / \partial x_{s}=a_{s} e_{s}, \theta^{i}=a_{i} d x_{i}$, $\theta^{s}=a_{s} d x_{s}$. Thus, the first and second fundamental forms of $\varphi$ are given by (1.11).

Definition 1.4. Let $\varphi: M^{n} \rightarrow \tilde{M}^{m}$ be an isometric immersion. Denote by $A_{\xi}$ the Weingarten endomorphism with respect to $\xi \in T^{\perp} M$. If for any $\xi \in T^{\perp} M$, the rank of $A_{\xi}$ is equal to $\min \{n, m-n\}$, then the normal bundle of $\varphi$ is said to be nondegenerate. 
Definition 1.5. Let $\left(M_{1}, g_{1}\right)$ and $\left(M_{2}, g_{2}\right)$ be two Riemannian manifolds, and $\varphi: M_{1} \times M_{2} \rightarrow \tilde{M}$ an isometric immersion of Riemannian product. If for a $\xi \in T^{\perp}\left(M_{1} \times M_{2}\right)$, there exist functions $\lambda_{1}$ and $\lambda_{2}$ such that $h_{\xi}=\lambda_{1} g_{1}+\lambda_{2} g_{2}$ where $h_{\xi}$ is the second fundamental tensor of $\varphi$ with respect to $\xi$, then $\varphi$ is called to be special quasiumbilical with respect to $\xi$. In particular, a special quasiumbilical immersion $\varphi$ with respect to $\xi \in T^{\perp}\left(M_{1} \times M_{2}\right)$ is umbilical when $\lambda_{1}=\lambda_{2}$.

In the case that $\varepsilon>0$, if $c \neq 0$, then we see from $(1.8)_{1-3}$ that rank $B=n$, which implies that $m-n \geq n-1$ and $\rho_{I}>0$ for all $I$. If $c=c_{1}=0$, then we see that $m-n \geq n_{2}-1$. In particular, when either $m-n=n-1$ for $c \neq 0$ or $m-n=n_{2}-1$ for $c=c_{1}=0$, then the normal bundle of $\varphi$ is nondegenerate and $\varphi$ satisfies one of the following conditions:

(i) $\varphi$ is not special quasiumbilical;

(ii) $\varphi$ is special quasiumbilical with respect to some $\xi \in T^{\perp}\left(M_{1} \times M_{2}\right)$, i.e., $h_{\xi}=\lambda_{1} g_{1}+\lambda_{2} g_{2}$, and $\lambda_{1} v_{1} \neq \lambda_{2} v_{2}$.

In the case that $\varepsilon<0$, if either $m-n<n-1$ for $c \neq 0$ or $m-n<n_{2}-1$ for $c=c_{1}=0$, then $\varphi$ is special quasiumbilical with respect to some $\xi \in$ $T^{\perp}\left(M_{1} \times M_{2}\right)$, satisfying $\lambda_{1} v_{1}=\lambda_{2} v_{2}$. Hence, if the immersion $\varphi$ satisfies one of (1.15), then we have either $m-n \geq n-1$ for $c \neq 0$ or $m-n \geq n_{2}-1$ for $c=c_{1}=0$.

For simplicity, in the following, we assume always that $m=2 n-1=$ $2\left(n_{1}+n_{2}\right)-1$. In such a case, $B$ of (1.9) is a non-degenerate $n \times n$ matrix, and $\rho_{I} \neq 0$ where only one of $\left\{\rho_{I}\right\}$ has the same sign as $\varepsilon$, and the remains are positive. Without loss of generality, suppose that $\rho_{\alpha}=\left(a_{\alpha}\right)^{-2}, \rho_{n}=\varepsilon\left(a_{n}\right)^{-2}$. On putting

$$
a_{I \alpha}=a_{I} b_{I \alpha}, \quad a_{i n}=v_{1} a_{i}, \quad a_{s n}=v_{2} a_{s}, \quad A=\left(a_{I J}\right),
$$

we see that $A$ satisfies $A \tilde{J}_{\varepsilon} A^{T}=\tilde{J}_{\varepsilon}$. So, by Lemma 1.3 , we have immediately

Proposition 1.6. Let $\varphi: M_{1}^{n_{1}}\left(c_{1}\right) \times M_{2}^{n_{2}}\left(c_{2}\right) \supset U \rightarrow M^{2 n-1}(c)$ be a locally isometric immersion with the flat normal bundle and the separable second fundamental form. If $\varphi$ satisfies one of (1.15), then there exist a line of curvature coordinates $\left(x_{i}, x_{s}\right)$ such that the first and second fundamental forms of $\varphi$ can be written as

$$
\begin{aligned}
& I=\sum_{i} a_{i}^{2} d x_{i}^{2}+\sum_{s} a_{s}^{2} d x_{s}^{2} \\
& I I=\sum_{\alpha}\left(\sum_{i} a_{i} a_{i \alpha} d x_{i}^{2}+\sum_{s} a_{s} a_{s \alpha} d x_{s}^{2}\right) e_{n+\alpha},
\end{aligned}
$$

and 


$$
A=\left(a_{I J}\right): \boldsymbol{R}^{n} \rightarrow S O_{\varepsilon}(n),
$$

where $a_{\text {in }}=v_{1} a_{i}, a_{s n}=v_{2} a_{s}$.

COROllary 1.7. Under the same hypothesis as in Proposition 1.5, if $c \neq 0$, then there exist a line of curvature spherical (hyperbolic) coordinates $\left\{x_{i}, x_{s}\right\}$ such that the first and second fundamental forms of $\varphi$ can be written as

$$
\begin{aligned}
& I=\sum_{i} a_{i}^{2} d x_{i}^{2}+\sum_{s} a_{s}^{2} d x_{s}^{2}, \\
& I I=\sum_{\alpha}\left(\sum_{i} v_{1} a_{i} a_{i \alpha} d x_{i}^{2}+\sum_{s} v_{2} a_{s} a_{s \alpha} d x_{s}^{2}\right) e_{n+\alpha},
\end{aligned}
$$

where $A=\left(a_{I J}\right): \boldsymbol{R}^{n} \rightarrow S O_{\varepsilon}(n), a_{i n}=a_{i}$ (resp. $\left.a_{s n}=a_{s}\right)$ are dependent only on $x_{i}$ (resp. $\left.x_{s}\right)$.

\section{§2. The zero-curvature condition}

Consider the Lie algebra

(2.1) $\quad \operatorname{so}_{e x}(m+3)=\left\{X \in \operatorname{sl}(m+3) \mid X J+J X^{T}=0\right\}, \quad J=\left(\begin{array}{cccc}\varepsilon_{1} & & & \\ & \varepsilon_{2} & & \\ & & I_{m} & \\ & & \varepsilon\end{array}\right)$.

The Lie group $S O_{e x}(m+3)$ corresponding to $s o_{e x}(m+3)$ is

$$
S O_{e x}(m+3)=\left\{A \in S L(m+3) \mid A J A^{T}=J\right\} .
$$

We now define a family of $s o_{e x}(m+3, C)$-valued 1 -forms parameterized by $\lambda \in \boldsymbol{C}^{*}=\boldsymbol{C} \backslash\{0\}$ as follows

$$
\tilde{\Theta}_{\lambda}=\left(\begin{array}{cccccc}
0 & 0 & -\varepsilon_{1} \kappa_{1} \theta_{1}^{T} & 0 & 0 & 0 \\
0 & 0 & 0 & -\varepsilon_{2} \kappa_{2} \theta_{2}^{T} & 0 & 0 \\
\kappa_{1} \theta_{1} & 0 & \omega_{1} & 0 & \lambda \beta_{1} & \lambda v_{1} \theta_{1} \\
0 & \kappa_{2} \theta_{2} & 0 & \omega_{2} & \lambda \beta_{2} & \lambda v_{2} \theta_{2} \\
0 & 0 & -\lambda \beta_{1}^{T} & -\lambda \beta_{2}^{T} & \eta & 0 \\
0 & 0 & -\lambda \varepsilon v_{1} \theta_{1}^{T} & -\lambda \varepsilon v_{2} \theta_{2}^{T} & 0 & 0
\end{array}\right) .
$$

LEMMA 2.1. There exists a locally isometric immersion $\varphi: M_{1}^{n_{1}}\left(c_{1}\right) \times$ $M_{2}^{n_{2}}\left(c_{2}\right) \supset U \rightarrow M^{m}(c)$ with flat normal bundle and the separable second fundamental form if and only if

$$
d \tilde{\Theta}_{\lambda}+\tilde{\Theta}_{\lambda} \wedge \tilde{\Theta}_{\lambda}=0
$$

for $\lambda \in C^{*}$. 
Proof. By (2.2), it is easy to see that (2.3) is equivalent to

$$
\begin{aligned}
& d \theta_{r}+\omega_{r} \wedge \theta_{r}=0, \\
& d \omega_{r}+\omega_{r} \wedge \omega_{r}-c_{r} \theta_{r} \wedge \theta_{r}^{T}=0, \\
& \beta_{r} \wedge \beta_{r}^{T}+\varepsilon v_{r}^{2} \theta_{r} \wedge \theta_{r}^{T}=0, \\
& \beta_{1} \wedge \beta_{2}^{T}+\varepsilon v_{1} v_{2} \theta_{1} \wedge \theta_{2}^{T}=0, \\
& d \eta+\eta \wedge \eta=0, \quad \theta_{r}^{T} \wedge \beta_{r}=0, \\
& \beta_{1}^{T} \wedge \beta_{1}+\beta_{2}^{T} \wedge \beta_{2}=0, \\
& d \beta_{r}+\beta_{r} \wedge \eta+\omega_{r} \wedge \beta_{r}=0 .
\end{aligned}
$$

On the other hand, since $\beta_{1}$ and $\beta_{2}$ may be expressed by $\theta_{1}$ and $\theta_{2}$, respectively, then $(1.2)_{6}$ is equivalent to $(2.4)_{6}$. Hence, (2.4) is equivalent to $(1.2) \sim(1.4)$.

In the following, we assume $m=2 n-1$. Under the same hypothesis as in Proposition 1.6, we set

$$
\begin{gathered}
f_{i j}=\frac{\partial_{j} a_{i}}{a_{j}} \quad(i \neq j), \quad f_{i i}=0, \quad f_{s t}=\frac{\partial_{t} a_{s}}{a_{t}} \quad(s \neq t), \quad f_{s s}=0, \\
b_{1}=\kappa_{1}\left(a_{1}, \ldots, a_{n_{1}}\right)^{T}, \quad b_{2}=\kappa_{2}\left(a_{n_{1}+1}, \ldots, a_{n}\right)^{T}, \quad b=\left(\begin{array}{l}
\kappa_{1}^{-1} v_{1} b_{1} \\
\kappa_{2}^{-1} v_{2} b_{2}
\end{array}\right), \\
\delta_{1}=\operatorname{diag}\left(d x_{1}, \ldots, d x_{n_{1}}\right), \quad \delta_{2}=\operatorname{diag}\left(d x_{n_{1}+1}, \ldots, d x_{n}\right), \\
A_{1}=\left(a_{i \alpha}\right), \quad A_{2}=\left(a_{s \alpha}\right), \quad F_{1}=\left(f_{i j}\right), \quad F_{2}=\left(f_{s t}\right) .
\end{gathered}
$$

We then see that

$$
b=A E_{n} \in S^{n-1}(\varepsilon), \quad F_{r} \in g l\left(n_{r}\right)_{*}=\left\{Y=\left(y_{i j}\right) \in g l\left(n_{r}\right) \mid y_{i i}=0\right\},
$$

where $E_{n}=\operatorname{diag}(\underbrace{0, \ldots, 0}, 1)$. Choose parallel frame fields in the normal bundle so that $\eta=0$. Thus, $\Xi$ of $(1.1)$ and $\tilde{\Theta}_{\lambda}$ of $(2.2)$ are reduced as

$$
\begin{gathered}
\text { (2.6) } \quad \boldsymbol{\Xi}=\left(\begin{array}{cccc}
0 & -c \kappa_{1}^{-1} b_{1}^{T} \delta_{1} & -c \kappa_{2}^{-1} b_{2}^{T} \delta_{2} & 0 \\
\kappa_{1}^{-1} \delta_{1} b_{1} & \omega_{1} & 0 & \delta_{1} A_{1} \\
\kappa_{2}^{-1} \delta_{2} b_{2} & 0 & \omega_{2} & \delta_{2} A_{2} \\
0 & -A_{1}^{T} \delta_{1} & -A_{2}^{T} \delta_{2} & 0
\end{array}\right), \\
\tilde{\Theta}_{\lambda}=\left(\begin{array}{cccccc}
0 & 0 & -\varepsilon_{1} b_{1}^{T} \delta_{1} & 0 & 0 & 0 \\
0 & 0 & 0 & -\varepsilon_{2} b_{2}^{T} \delta_{2} & 0 & 0 \\
\delta_{1} b_{1} & 0 & \omega_{1} & 0 & \lambda \delta_{1} A_{1} & \lambda \kappa_{1}^{-1} v_{1} \delta_{1} b_{1} \\
0 & \delta_{2} b_{2} & 0 & \omega_{2} & \lambda \delta_{2} A_{2} & \lambda \kappa_{2}^{-1} v_{2} \delta_{2} b_{2} \\
0 & 0 & -\lambda A_{1}^{T} \delta_{1} & -\lambda A_{2}^{T} \delta_{2} & 0 & 0 \\
0 & 0 & -\lambda \varepsilon \kappa_{1}^{-1} v_{1} b_{1}^{T} \delta_{1} & -\lambda \varepsilon \kappa_{2}^{-1} v_{2} b_{2}^{T} \delta_{2} & 0 & 0
\end{array}\right) .
\end{gathered}
$$


Lemma 2.2. Let $h: \boldsymbol{R}^{n} \rightarrow \boldsymbol{R}^{2 n+2}$ satisfy the equation $d h=h \tilde{\Theta}_{1}$. Write $h$ as a row vector

$$
\begin{array}{cccccc}
1 & 1 & n_{1} & n_{2} & n-1 & 1 \\
\left(\xi_{1},\right. & \xi_{2}, & \eta_{1}, & \eta_{2}, & \zeta, & \tilde{\zeta}),
\end{array}
$$

where $\tilde{\zeta}$ satisfies that $\tilde{\zeta}(0)=\kappa_{1}^{-1} v_{1} \xi_{1}(0)+\kappa_{2}^{-1} v_{2} \xi_{2}(0)$. Then $\tilde{h}=\left(\xi, \eta_{1}, \eta_{2}, \zeta\right)$ : $\boldsymbol{R}^{n} \rightarrow \boldsymbol{R}^{2 n}$ satisfies the equation $d \tilde{h}=\tilde{h} \Xi$, where $\xi=\kappa_{1}^{-1} \xi_{1}+\kappa_{2}^{-1} \xi_{2}$.

Proof. Since $h$ satisfies $d h=h \tilde{\Theta}_{1}$, then we have from (2.7)

$$
\begin{aligned}
d \xi_{r} & =\eta_{r} \delta_{r} b_{r}, \\
d \eta_{r} & =\eta_{r} \omega_{r}-\zeta A_{r}^{T} \delta_{r}-\left(\varepsilon_{r} \xi_{r}+\varepsilon \kappa_{r}^{-1} v_{r} \tilde{\zeta}\right) b_{r}^{T} \delta_{r}, \\
d \zeta & =\eta_{1} \delta_{1} A_{1}+\eta_{2} \delta_{2} A_{2}, \\
d \tilde{\zeta} & =\kappa_{1}^{-1} v_{1} \eta_{1} \delta_{1} b_{1}+\kappa_{2}^{-1} v_{2} \eta_{2} \delta_{2} b_{2}=\kappa_{1}^{-1} v_{1} d \xi_{1}+\kappa_{2}^{-1} v_{2} d \xi_{2} .
\end{aligned}
$$

By the last equation and the initial condition, we see that

$$
\tilde{\zeta}=\kappa_{1}^{-1} v_{1} \xi_{1}+\kappa_{2}^{-1} v_{2} \xi_{2} \text {. }
$$

It follows that

$$
\begin{aligned}
\varepsilon_{1} \xi_{1}+\varepsilon \kappa_{1}^{-1} v_{1} \tilde{\zeta} & =\kappa_{1}^{-1}\left\{\left(\kappa_{1} \varepsilon_{1}+\varepsilon v_{1}^{2} \kappa_{1}^{-1}\right) \xi_{1}+\varepsilon v_{1} v_{2} \kappa_{2}^{-1} \xi_{2}\right\} \\
& =c \kappa_{1}^{-1}\left(\kappa_{1}^{-1} \xi_{1}+\kappa_{2}^{-1} \xi_{2}\right)=c \kappa_{1}^{-1} \xi .
\end{aligned}
$$

In the similar way, we can obtain $\varepsilon_{2} \xi_{2}+\varepsilon \kappa_{2}^{-1} v_{2} \tilde{\zeta}=c \kappa_{2}^{-1} \xi$. Hence, we have

$$
\begin{aligned}
d \xi & =\kappa_{1}^{-1} \eta_{1} \delta_{1} b_{1}+\kappa_{2}^{-1} \eta_{2} \delta_{2} b_{2}, \\
d \eta_{r} & =\eta_{r} \omega_{r}-\zeta A_{r}^{T} \delta_{r}-c \kappa_{r}^{-1} \xi b_{r}^{T} \delta_{r}, \\
d \zeta & =\eta_{1} \delta_{1} A_{1}+\eta_{2} \delta_{2} A_{2},
\end{aligned}
$$

i.e., $\tilde{h}=\left(\xi, \eta_{1}, \eta_{2}, \zeta\right)$ satisfies $d \tilde{h}=\tilde{h} \Xi$.

For simplicity, we write a $p \times(2 n+2)$ matrix $\mathscr{M}$ as a row matrix

$$
\begin{array}{cccccc}
1 & 1 & n_{1} & n_{2} & n_{1} & n_{2} \\
\left(\mathscr{M}^{(1)}\right. & \mathscr{M}^{(2)} & \mathscr{M}^{(3)} & \mathscr{M}^{(4)} & \mathscr{M}^{(5)} & \left.\mathscr{M}^{(6)}\right) .
\end{array}
$$

Particularly, we write a $(2 n+2) \times(2 n+2)$ matrix $\mathscr{M}$ as a block matrix

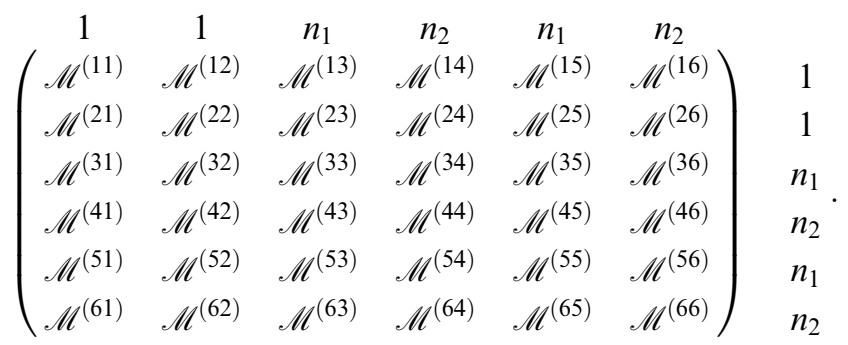


By using the guage transformation

$$
\Theta_{\lambda}=H \tilde{\Theta}_{\lambda} H^{-1}-d H H^{-1}, \quad \text { where } H=\left(\begin{array}{cc}
I_{n+2} & 0 \\
0 & A
\end{array}\right) \in S O_{e x}(2 n+2),
$$

we obtain

$$
\Theta_{\lambda}=\left(\begin{array}{cccccc}
0 & 0 & -\varepsilon_{1} b_{1}^{T} \delta_{1} & 0 & 0 & 0 \\
0 & 0 & 0 & -\varepsilon_{2} b_{2}^{T} \delta_{2} & 0 & 0 \\
\delta_{1} b_{1} & 0 & \omega_{1} & 0 & \lambda \delta_{1} & 0 \\
0 & \delta_{2} b_{2} & 0 & \omega_{2} & 0 & \lambda \delta_{2} \\
0 & 0 & -\lambda \delta_{1} & 0 & \vartheta_{1} & 0 \\
0 & 0 & 0 & -\lambda J_{\varepsilon} \delta_{2} & 0 & \vartheta_{2}
\end{array}\right)
$$

where

$$
\begin{gathered}
\omega_{r}=\delta_{r} F_{r}-F_{r}^{T} \delta_{r}, \quad \vartheta_{1}=\delta_{1} F_{1}^{T}-F_{1} \delta_{1}, \quad \vartheta_{2}=J_{\varepsilon} \delta_{2} F_{2}^{T} J_{\varepsilon}-F_{2} \delta_{2}, \\
J_{\varepsilon}=\left(\begin{array}{cc}
I_{n_{2}-1} & 0 \\
0 & \varepsilon
\end{array}\right) .
\end{gathered}
$$

It is easy to see that $d \Theta_{\lambda}+\Theta_{\lambda} \wedge \Theta_{\lambda}=0$ if and only if $d \tilde{\Theta}_{\lambda}+\tilde{\Theta}_{\lambda} \wedge \tilde{\Theta}_{\lambda}=0$, which is equivalent to that $\left(F_{1}, F_{2}, A\right)$ satisfies the following system of PDE:

$$
\left\{\begin{array}{l}
d A_{r}=-\vartheta_{r} A_{r}, \\
d b_{r}=-\vartheta_{r} b_{r}, \\
d \omega_{r}+\omega_{r} \wedge \omega_{r}-\varepsilon_{r} \delta_{r} b_{r} \wedge b_{r}^{T} \delta_{r}=0,
\end{array}\right.
$$

i.e., the Gauss-Codazzi-Ricci equations for the isometric immersion $\varphi$.

On putting

$a=\left(\begin{array}{cccccc}0 & 0 & 0 & 0 & 0 & 0 \\ 0 & 0 & 0 & 0 & 0 & 0 \\ 0 & 0 & 0 & 0 & \delta_{1} & 0 \\ 0 & 0 & 0 & 0 & 0 & \delta_{2} \\ 0 & 0 & -\delta_{1} & 0 & 0 & 0 \\ 0 & 0 & 0 & -\delta_{2} & 0 & 0\end{array}\right), \quad v=\left(\begin{array}{cccccc}0 & 0 & 0 & 0 & -\varepsilon_{1} b_{1}^{T} & 0 \\ 0 & 0 & 0 & 0 & 0 & -\varepsilon_{2} b_{2}^{T} J_{\varepsilon} \\ 0 & 0 & 0 & 0 & -F_{1}^{T} & 0 \\ 0 & 0 & 0 & 0 & 0 & -F_{2}^{T} J_{\varepsilon} \\ b_{1} & 0 & F_{1} & 0 & 0 & 0 \\ 0 & b_{2} & 0 & F_{2} & 0 & 0\end{array}\right)$,

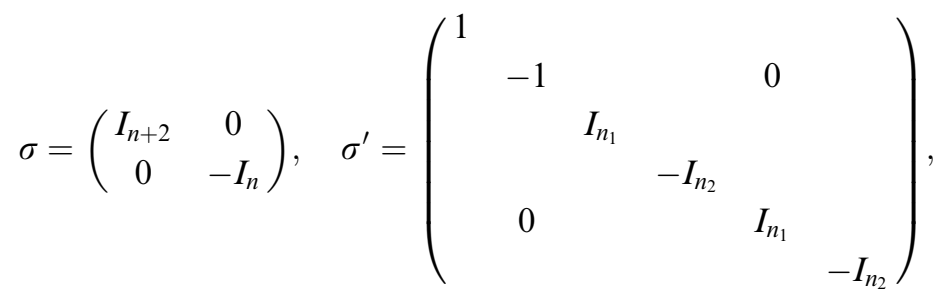

we have 


$$
\begin{gathered}
\Theta_{\lambda}=a \lambda+[a, v], \\
b_{1}=v^{(51)}, \quad b_{2}=v^{(62)}, \quad F_{1}=v^{(53)}, \quad F_{2}=v^{(64)} .
\end{gathered}
$$

With respect to $\sigma$ and $\sigma^{\prime}$, the Lie algebra $\mathscr{G}=s o_{e x}(2 n+2)$ has the Cartan decompositions $\mathscr{G}=\mathscr{P} \oplus \mathscr{K}=\mathscr{P}^{\prime} \oplus \mathscr{K}^{\prime}$, respectively. Let

$$
\begin{gathered}
\mathscr{G}_{a}=\{y \in \mathscr{G} \mid[a, y]=0\}, \quad \mathscr{G}_{a}^{\perp}=\left\{z \in \mathscr{G} \mid \operatorname{tr}(z y)=0 \text { for } y \in \mathscr{G}_{a}\right\}, \\
\wedge_{\sigma, \sigma^{\prime}} \mathscr{G}=\left\{X(\lambda) \in \wedge \mathscr{G} \mid \sigma X(\lambda) \sigma=X(-\lambda), \sigma^{\prime} X(\lambda) \sigma^{\prime}=X(\lambda)\right\} .
\end{gathered}
$$

Clearly, $a$ is $\left(\mathscr{P} \cap \mathscr{K}^{\prime}\right)$-valued 1 -form, $v: U \rightarrow g_{a}^{\perp} \cap \mathscr{P} \cap \mathscr{K}^{\prime}$ is a smooth map. Thus, $\Theta_{\lambda}$ is a $\wedge_{\sigma, \sigma^{\prime}} \mathscr{G}$-valued 1-form.

Consider the system

$$
\left\{\begin{array}{l}
d \Phi_{\lambda}=\Phi_{\lambda}(a \lambda+[a, v])=\Phi_{\lambda} \Theta_{\lambda} \\
\Phi_{\lambda}(0)=I_{2 n+2}
\end{array}\right.
$$

of which the integrability condition is (2.11). For the solution $\Phi_{\lambda}$ to (2.14), we have

$$
A=\left(\begin{array}{cc}
\Phi_{0}^{(55)} & \Phi_{0}^{(56)} J_{\varepsilon} \\
J_{\varepsilon} \Phi_{0}^{(65)} & J_{\varepsilon} \Phi_{0}^{(66)} J_{\varepsilon}
\end{array}\right)^{T} A(0)
$$

Set $\tilde{\Phi}_{\lambda}=Q_{1} H^{-1}(0) \Phi_{\lambda} H$, where

$$
Q_{1}=\left(\begin{array}{ccccc}
1 & 0 & 0 & 0 & \kappa_{2}^{-1} v_{2} \\
0 & 1 & 0 & 0 & \kappa_{1}^{-1} v_{1} \\
0 & 0 & I_{n} & 0 & 0 \\
0 & 0 & 0 & A_{2}(0) & 0 \\
0 & 0 & 0 & A_{1}(0) & 0
\end{array}\right) .
$$

Then $\tilde{\Phi}_{\lambda}$ satisfies

$$
d \tilde{\Phi}_{\lambda}=\tilde{\Phi}_{\lambda} \tilde{\Theta}_{\lambda}
$$

Write

$$
\tilde{\Phi}_{1}=\left(\mathbf{r}_{1}, \mathbf{r}_{2}, \tilde{e}_{1}, \ldots, \tilde{e}_{2 n}\right), \quad \tilde{\mathbf{r}}=\frac{1}{\kappa_{1}} \mathbf{r}_{1}+\frac{1}{\kappa_{2}} \mathbf{r}_{2}, \quad \tilde{\Psi}=\left(\tilde{\mathbf{r}}, \tilde{e}_{1}, \ldots, \tilde{e}_{2 n-1}\right) .
$$

By a straightforward calculation, one can see that $\tilde{e}_{2 n}(0)=v_{1} \kappa_{1}^{-1} \mathbf{r}_{1}(0)+$ $v_{2} \kappa_{2}^{-1} \mathbf{r}_{2}(0)$. So, it follows from Lemma 2.2 that $\tilde{\Psi}$ satisfies the following system

$$
d \tilde{\Psi}=\tilde{\Psi} \Xi, \quad \tilde{\Psi}(0)=\left(\begin{array}{ccccc}
\kappa_{1}^{-1} & \kappa_{2}^{-1} & 0 & 0 & 0 \\
0 & 0 & I_{n} & 0 & 0 \\
0 & 0 & 0 & A_{1}(0)^{T} & A_{2}(0)^{T}
\end{array}\right)^{T}
$$

where $\Xi$ is defined in (2.6). Set 


$$
\Psi=Q_{2} \tilde{\Psi} \quad \text { with } Q_{2}=\left(\begin{array}{ccccc}
\frac{1}{2} \kappa_{1} & \frac{1}{2} \kappa_{2} & 0 & 0 & 0 \\
0 & 0 & I_{n} & 0 & 0 \\
0 & 0 & 0 & A_{1}(0)^{T} & A_{2}(0)^{T} J_{\varepsilon}
\end{array}\right) .
$$

Then $\Psi: U \rightarrow S O_{c}(2 n)$ satisfies the system (1.1). Hence, we obtain

where

$$
\begin{aligned}
\mathbf{r} & =J^{2} Q_{2} \tilde{\mathbf{r}}=J^{2} Q_{2} Q_{1} H^{-1}(0) \Phi_{1}\left(\kappa_{1}^{-1} H^{(1)}+\kappa_{2}^{-1} H^{(2)}\right) \\
& =Q\left(\kappa_{1}^{-1} \Phi_{1}^{(1)}+\kappa_{2}^{-1} \Phi_{1}^{(2)}\right),
\end{aligned}
$$

$$
Q=\left(\begin{array}{ccccc}
\frac{c^{2}}{2} \kappa_{1} & \frac{c^{2}}{2} \kappa_{2} & 0 & \frac{c\left(2-c c_{1}\right)}{2 \kappa_{1}} b_{1}^{T}(0) & \frac{c\left(2-c c_{2}\right)}{2 \kappa_{2}} b_{2}^{T}(0) J_{\varepsilon} \\
0 & 0 & I_{n} & 0 & 0 \\
0 & 0 & 0 & A_{1}(0)^{T} & A_{2}(0)^{T} J_{\varepsilon}
\end{array}\right)
$$

is a constant $2 n \times(2 n+2)$ matrix. Summing up and combining Proposition 1.6, we have proved the following

THEOREM 2.3. Let $U \subset M_{1}^{n_{1}}\left(c_{1}\right) \times M_{2}^{n_{2}}\left(c_{2}\right)$ be a simply-connected domain around the origin $x=0$, and $\varphi: U \rightarrow M^{2 n-1}(c), n=n_{1}+n_{2}$, a locally isometric immersion, of which the first and second fundamental forms can be written as (1.16). Then there exists a smooth map $\left(F_{1}, F_{2}, b\right): U \rightarrow \operatorname{gl}\left(n_{1}\right)_{*} \times g l\left(n_{2}\right)_{*} \times$ $S^{n-1}(\varepsilon)$ such that $\Theta_{\lambda}$ defined by (2.9) is a flat connection and the system (2.14) has a unique solution $\Phi_{\lambda}$ satisfying

$$
\mathbf{r}=\mathbf{r}_{c} \circ \varphi=Q\left(\kappa_{1}^{-1} \Phi_{1}^{(1)}+\kappa_{2}^{-1} \Phi_{1}^{(2)}\right) .
$$

Conversely, for a map $\left(F_{1}, F_{2}, b\right): \boldsymbol{R}^{n} \rightarrow g l\left(n_{1}\right)_{*} \times g l\left(n_{2}\right)_{*} \times S^{n-1}(\varepsilon)$, if (2.14) has a unique solution $\Phi_{\lambda}$, then there exists a smooth map $A=\left(a_{i j}\right): U \rightarrow O_{\varepsilon}(n)$ such that $b=A E_{n}$. Moreover, if $U=\left\{x \in \boldsymbol{R}^{n} \mid a_{i}(x) \neq 0, a_{s}(x) \neq 0\right.$ for all $\left.i, s\right\}$ is not empty, then there exists an isometric immersion $\varphi: U \rightarrow M^{2 n-1}(c)$ with flat normal bundle such that the first and second fundamental forms of $\varphi$ are given by (1.16), and (2.18) holds.

\section{§3. Darboux transformation}

We now consider the Darboux transformation for solutions of the system (2.14). Since $\Theta_{\lambda}=a \lambda+[a, u]$ is a $\wedge_{\sigma, \sigma^{\prime}} \mathscr{G}$-valued 1-form, then $\Phi_{\lambda}$ satisfies the following $K^{\prime} /\left(K \cap K^{\prime}\right)$-reality condition (cf. [TU]):

$$
f(\lambda) J f(\bar{\lambda})^{*}=J, \quad \overline{f(\bar{\lambda})}=f(\lambda), \quad \sigma f(\lambda) \sigma=f(-\lambda), \quad \sigma^{\prime} f(\lambda) \sigma^{\prime}=f(\lambda) .
$$

Let $O_{\infty}$ be an open neighborhood around $\infty$ in $C \cup\{\infty\}=S^{2}$, and let

$$
\begin{gathered}
G_{-}^{m}=\left\{f: O_{\infty} \rightarrow G L(N, C) \mid f\right. \text { is a holomorphic rational fraction } \\
\text { satisfying } \left.(3.1)_{1} \text { and } f(\infty)=I_{N}\right\}, \\
\left(G_{-}^{m}\right)_{\sigma, \sigma^{\prime}}=\left\{f(\lambda) \in G_{-}^{m} \mid f(\lambda) \text { satisfies }(3.1)\right\} .
\end{gathered}
$$


A map $\pi: V \rightarrow C^{N}$ is called a $J$-Hermitian projection if

$$
\pi^{2}=\pi, \quad J \pi^{*}=\pi J .
$$

Clearly, $\pi^{\prime}=I-\pi$ is also a $J$-Hermitian projection if $\pi$ is one. Thus, a simple element of $G_{-}^{m}$ is of the form [TU]:

$$
h_{\alpha, \pi}(\lambda)=\pi^{\prime}+\frac{\lambda-\alpha}{\lambda-\bar{\alpha}} \pi=I-\frac{\alpha-\bar{\alpha}}{\lambda-\bar{\alpha}} \pi
$$

for $\alpha \in C^{*}=C \backslash\{0\}$. Let $\tau$ be a diagonal complex matrix satisfying $\tau^{2}=\sigma$. A direct computation yields the following

Lemma 3.1. Let $\pi_{0}$ be a J-Hermitian projection in $C^{N}$ satisfying

$$
\bar{\pi}_{0}=\pi_{0}, \quad \sigma \pi_{0} \sigma \pi_{0}=\pi_{0} \sigma \pi_{0} \sigma, \quad \sigma^{\prime} \pi_{0} \sigma^{\prime}=\pi_{0} .
$$

If $\pi=\tau^{-1} \pi_{0} \tau$, then $f(\lambda)=h_{\alpha, \pi} h_{-\alpha, \sigma \pi \sigma} \in\left(G_{-}^{m}\right)_{\sigma, \sigma^{\prime}}$ for $\alpha \in \sqrt{-1} \boldsymbol{R}$.

Let $\Phi_{\lambda}$ be a solution to (2.14), $L$ a constant complex $s \times N$ matrix satisfying

$$
L J \sigma L^{T}=0, \quad \operatorname{span}\left\{L \sigma^{\prime}\right\}=\operatorname{span}\{L\}, \quad \operatorname{det}\left(L J L^{T}\right) \neq 0 .
$$

Set

$$
\begin{gathered}
\pi_{0}=J L^{T}\left(L J L^{T}\right)^{-1} L, \quad \pi=\tau^{-1} \pi_{0} \tau, \\
h=L \tau \Phi_{\alpha}, \quad \tilde{\pi}_{1}=J h^{*}\left(h J h^{*}\right)^{-1} h, \\
\Psi_{\lambda}=h_{\bar{\alpha}, \pi} \Phi_{\lambda} h_{\alpha, \tilde{\pi}_{1}}, \quad \tilde{h}=L \tau \sigma \Psi_{-\alpha}, \quad \tilde{\pi}_{2}=J \tilde{h}^{*}\left(\tilde{h} J \tilde{h}^{*}\right)^{-1} \tilde{h} .
\end{gathered}
$$

Let

$$
\begin{gathered}
\alpha=\sqrt{-1} \mu \quad \text { for } \mu \in \boldsymbol{R} \backslash\{0\}, \\
\Delta=\frac{1}{2} h J h^{*}, \quad \tilde{\Delta}=\frac{1}{2} \tilde{h} J \tilde{h}^{*}, \quad h^{\prime}=\frac{d h}{d \mu}, \quad \rho=-h^{\prime} \sigma \tau h^{*} .
\end{gathered}
$$

Then it is easy to see that $\pi_{0}$ satisfies conditions in Lemma 3.1, $\rho$ is a real skewsymmetric $s \times s$ matrix, $\Delta$ and $\tilde{\Delta}$ all are real symmetric invertible $s \times s$ matrices. Hence, we may write the following Darboux matrix [HS]:

$$
\begin{aligned}
D_{\lambda}= & h_{\sqrt{-1} \mu, \tilde{\pi}_{1}} h_{-\sqrt{-1} \mu, \tilde{\pi}_{2}}=\left(I-\frac{2 \sqrt{-1} \mu}{\lambda+\sqrt{-1} \mu} \tilde{\pi}_{1}\right)\left(I+\frac{2 \sqrt{-1} \mu}{\lambda-\sqrt{-1} \mu} \tilde{\pi}_{2}\right) \\
= & I-\frac{\sqrt{-1} \mu}{\lambda+\sqrt{-1} \mu} J h^{*} \Delta^{-1} h \\
& +\frac{\sqrt{-1} \mu}{\lambda-\sqrt{-1} \mu} J\left(\sigma h^{*}+\mu h^{*} \Delta^{-1} \rho\right) \tilde{\Delta}^{-1}\left(h \sigma-\mu \rho \Delta^{-1} h\right) \\
& +\frac{2 \mu^{3}}{\lambda^{2}+\mu^{2}} J h^{*} \Delta^{-1} \rho \tilde{\Delta}^{-1}\left(h \sigma-\mu \rho \Delta^{-1} h\right),
\end{aligned}
$$




$$
\begin{aligned}
d_{1} & =\left.\left(\frac{d D_{\lambda^{-1}}}{d \lambda}\right)\right|_{\lambda=0}=2 \sqrt{-1} \mu\left(\tilde{\pi}_{2}^{\perp}-\tilde{\pi}_{1}^{\perp}\right) \\
& =\sqrt{-1} \mu J\left\{\left(\sigma h^{*}+\mu h^{*} \Delta^{-1} \rho\right) \tilde{\Delta}^{-1}\left(h \sigma-\mu \rho \Delta^{-1} h\right)-h^{*} \Delta^{-1} h\right\} .
\end{aligned}
$$

Thus, in the similar way as in [HS], we have following

THEOREM 3.2. Let $\Phi_{\lambda}$ be a solution of the system (2.14), and $L$ a real constant $s \times N$ matrix. Set $h=L \tau \Phi_{\sqrt{-1} \mu}$ for $\mu \in \boldsymbol{R} \backslash\{0\}$. Then there is an open neighbourhood $U$ around the origin 0 such that on $U, \tilde{\Phi}_{\lambda}=D_{\lambda}(0)^{-1} \Phi_{\lambda} D_{\lambda}$ satisfies the system (2.14) with $\tilde{v}=v+\left(d_{1}\right)_{\mathscr{G} \perp}: U \rightarrow \mathscr{G}_{a}^{\perp} \cap \mathscr{P} \cap \mathscr{K}^{\prime}$, namely, a $\lambda+[a, \tilde{v}]$ is a $\wedge_{\sigma, \sigma^{\prime}} \mathscr{G}$-valued 1-form, where $D_{\lambda}$ and $d_{1}$ are defined by (3.4) and (3.5), respectively.

In the following, we take $N=2(n+1)$. By Theorem 2.3 and Theorem 3.2, it is sufficient to find the Darboux matrix (3.4) preserving $b(x) \in S^{n-1}(\varepsilon)$.

LeMma 3.3. Let $\Phi_{\lambda}$ be a solution of the system (2.14), L a complex constant $s \times 2(n+1)$ matrix. Assume that $\lambda_{0} \in C$ and $h=L \Phi_{\lambda_{0}}=\left(\xi_{1}, \xi_{2}, \eta_{1}, \eta_{2}, \zeta_{1}, \zeta_{2}\right)$. Then $d\left(\zeta_{r} b_{r}-\lambda_{0} \xi_{r}\right)=0$.

Proof. Since $h$ satisfies the equation $d h=h \Theta_{\lambda_{0}}$, i.e.,

$$
\begin{aligned}
& d \xi_{r}=\eta_{r} \delta_{r} b_{r}, \\
& d \eta_{1}=-\varepsilon_{1} \xi_{1} b_{1}^{T} \delta_{1}+\eta_{1} \omega_{1}-\lambda_{0} \zeta_{1} \delta_{1}, \\
& d \eta_{2}=-\varepsilon_{2} \xi_{2} b_{2}^{T} \delta_{2}+\eta_{2} \omega_{2}-\lambda_{0} J_{\varepsilon} \zeta_{2} \delta_{2}, \\
& d \zeta_{r}=\lambda_{0} \eta_{r} \delta_{r}+\zeta_{r} \vartheta_{r},
\end{aligned}
$$

then, by (2.11), we get

$$
\begin{aligned}
d\left(\zeta_{r} b_{r}-\lambda_{0} \xi_{r}\right) & =\left(d \zeta_{r}\right) b_{r}+\zeta_{r} d b_{r}-\lambda_{0} d \xi_{r} \\
& =\left(\lambda_{0} \eta_{r} \delta_{r}+\zeta_{r} \vartheta_{r}\right) b_{r}-\zeta_{r} \vartheta_{r} b_{r}-\lambda_{0} \eta_{r} \delta_{r} b_{r}=0 .
\end{aligned}
$$

Let $\mu \in \boldsymbol{R} \backslash\{0\}, h=L \tau \Phi_{\sqrt{-1} \mu}=\left(\xi_{1}, \xi_{2}, \eta_{1}, \eta_{2}, \sqrt{-1} \zeta_{1}, \sqrt{-1} \zeta_{2}\right)$ satisfying

$$
\begin{aligned}
& d \xi_{r}=\eta_{r} \delta_{r} b_{r}, \\
& d \eta_{1}=-\varepsilon_{1} \xi_{1} b_{1}^{T} \delta_{1}+\eta_{1} \omega_{1}+\mu \zeta_{1} \delta_{1}, \\
& d \eta_{2}=-\varepsilon_{2} \xi_{2} b_{2}^{T} \delta_{2}+\eta_{2} \omega_{2}+\mu \zeta_{2} J_{\varepsilon} \delta_{2}, \\
& d \zeta_{r}=\mu \eta_{r} \delta_{r}+\zeta_{r} \vartheta_{r} .
\end{aligned}
$$

By Theorem 3.2 and Lemma 3.3, if we choose $L$ such that $L$ satisfies (3.3) and

$$
L^{(5)} b_{1}(0)^{T}-\mu L^{(1)}=0, \quad L^{(6)} b_{2}(0)^{T}-\mu L^{(2)}=0,
$$

then there exists an open neighbourhood $U$ around the origin 0 such that $h=$ $\left(\xi_{1}, \xi_{2}, \eta_{1}, \eta_{2}, \sqrt{-1} \zeta_{1}, \sqrt{-1} \zeta_{2}\right)$ satisfies 


$$
\begin{gathered}
h J \sigma h^{*}=\varepsilon_{1} \xi_{1} \xi_{1}^{T}+\varepsilon_{2} \xi_{2} \xi_{2}^{T}+\eta_{1} \eta_{1}^{T}+\eta_{2} \eta_{2}^{T}-\zeta_{1} \zeta_{1}^{T}-\zeta_{2} J_{\varepsilon} \zeta_{2}^{T}=0, \\
\operatorname{det}\left(h J h^{*}\right) \neq 0, \quad \zeta_{r} b_{r}^{T}-\mu \xi_{r}=0, \\
\operatorname{span}\left\{h \sigma^{\prime}\right\}=\operatorname{span}\{h\},
\end{gathered}
$$

in $U$. Thus, (3.4) can be written as

$D_{\lambda}=I-\frac{2 \mu}{\lambda^{2}+\mu^{2}}$

$\left(\begin{array}{cccccc}\mu \varepsilon_{1} \xi_{1}^{T} \Delta_{1} \xi_{1} & 0 & \mu \varepsilon_{1} \xi_{1}^{T} \Delta_{1} \eta_{1} & 0 & -\lambda \varepsilon_{1} \xi_{1}^{T} \Delta_{2} \zeta_{1} & 0 \\ 0 & \mu \varepsilon_{2} \xi_{2}^{T} \Delta_{1} \xi_{2} & 0 & \mu \varepsilon_{2} \xi_{2}^{T} \Delta_{1} \eta_{2} & 0 & -\lambda \varepsilon_{2} \xi_{2}^{T} \Delta_{2} \zeta_{2} \\ \mu \eta_{1}^{T} \Delta_{1} \xi_{1} & 0 & \mu \eta_{1}^{T} \Delta_{1} \eta_{1} & 0 & -\lambda \eta_{1}^{T} \Delta_{2} \zeta_{1} & 0 \\ 0 & \mu \eta_{2}^{T} \Delta_{1} \xi_{2} & 0 & \mu \eta_{2}^{T} \Delta_{1} \eta_{2} & 0 & -\lambda \eta_{2}^{T} \Delta_{2} \zeta_{2} \\ \lambda \zeta_{1}^{T} \Delta_{1} \xi_{1} & 0 & \lambda \zeta_{1}^{T} \Delta_{1} \eta_{1} & 0 & \mu \zeta_{1}^{T} \Delta_{2} \zeta_{1} & 0 \\ 0 & \lambda J_{\varepsilon} \zeta_{2}^{T} \Delta_{1} \xi_{2} & 0 & \lambda J_{\varepsilon} \zeta_{2}^{T} \Delta_{1} \eta_{2} & 0 & \mu J_{\varepsilon} \zeta_{2}^{T} \Delta_{2} \zeta_{2}\end{array}\right)$,

where

$$
\begin{aligned}
& \Delta_{1}=(\Delta+\mu \rho)^{-1}, \quad \Delta_{2}=(\Delta-\mu \rho)^{-1} \\
& \Delta=\frac{1}{2} h J h^{*}=\zeta_{1} \zeta_{1}^{T}+\zeta_{2} J_{\varepsilon} \zeta_{2}^{T}=\varepsilon_{1} \xi_{1} \xi_{1}^{T}+\varepsilon_{2} \xi_{2} \xi_{2}^{T}+\eta_{1} \eta_{1}^{T}+\eta_{2} \eta_{2}^{T}, \\
& \rho=-h^{\prime} \sigma J h^{*}=\zeta_{1}^{\prime} \zeta_{1}^{T}+\zeta_{2}^{\prime} J_{\varepsilon} \zeta_{2}^{T}-\varepsilon_{1} \xi_{1}^{\prime} \xi_{1}^{T}-\varepsilon_{2} \xi_{2}^{\prime} \xi_{2}^{T}-\eta_{1}^{\prime} \eta_{1}^{T}-\eta_{2}^{\prime} \eta_{2}^{T} .
\end{aligned}
$$

Here $h^{\prime}=d h / d \mu=\left(\xi_{1}^{\prime}, \xi_{2}^{\prime}, \eta_{1}^{\prime}, \eta_{2}^{\prime}, \sqrt{-1} \zeta_{1}^{\prime}, \sqrt{-1} \zeta_{2}^{\prime}\right)$.

Set $\tilde{\Phi}_{\lambda}=\Phi_{\lambda} D_{\lambda}$. It is easy from Theorem 3.2 to see that $\tilde{\Phi}_{\lambda}$ satisfies the system (2.14) with $\tilde{v}=v+\left(d_{1}\right)_{\mathscr{G}_{a}^{\perp}}$. Thus, by (2.13) and (2.15), we have

$$
\begin{gathered}
\tilde{F}_{1}=\left(\tilde{v}^{(53)}\right)_{\mathrm{off}}=F_{1}-2 \mu\left(\zeta_{1}^{T} \Delta_{1} \eta_{1}\right)_{\mathrm{off}}, \\
\tilde{F}_{2}=\left(\tilde{v}^{(64)}\right)_{\mathrm{off}}=F_{2}-2 \mu\left(J_{\varepsilon} \zeta_{2}^{T} \Delta_{1} \eta_{2}\right)_{\mathrm{off}}, \\
\tilde{b}_{1}=\tilde{v}^{(51)}=b_{1}-2 \mu \zeta_{1}^{T} \Delta_{1} \xi_{1}=\kappa_{1}^{-1}\left(\tilde{a}_{1}, \ldots, \tilde{a}_{n_{1}}\right)^{T}, \\
\tilde{b}_{2}=\tilde{v}^{(64)}=b_{2}-2 \mu J_{\varepsilon} \zeta_{2}^{T} \Delta_{1} \xi_{2}=\kappa_{2}^{-1}\left(\tilde{a}_{n_{1}+1}, \ldots, \tilde{a}_{n}\right)^{T}, \\
\tilde{A}=\left(\begin{array}{ccc}
\tilde{\Phi}_{0}^{(55)} & \tilde{\Phi}_{0}^{(56)} J_{\varepsilon} \\
J_{\varepsilon} \tilde{\Phi}_{0}^{(65)} & J_{\varepsilon} \tilde{\Phi}_{0}^{(66)} J_{\varepsilon}
\end{array}\right)^{T} A(0)=A-2\left(\begin{array}{cc}
\zeta_{1}^{T} \Delta_{1} \zeta_{1} & 0 \\
0 & J_{\varepsilon} \zeta_{2}^{T} \Delta_{1} \zeta_{2}
\end{array}\right) A .
\end{gathered}
$$

Since $\zeta_{r} b_{r}=\mu \xi_{r}$, then $\tilde{b}^{T}=\left(\kappa_{1}^{-1} v_{1} \tilde{b}_{1}^{T}, \kappa_{2}^{-1} v_{2} \tilde{b}_{2}^{T}\right)=\tilde{A} E_{n}$. Noting that $\tilde{A} \in O_{\varepsilon}(n)$, we see that $\tilde{b} \in S^{n-1}(\varepsilon)$. Hence, we have the following

THEOREM 3.4. Let $\varphi: M_{1}^{n_{1}}\left(c_{1}\right) \times M_{2}^{n_{2}}\left(c_{2}\right) \rightarrow M^{2 n-1}(c), n=n_{1}+n_{2}$, be a local isometric immersion whose first and second fundamental forms can be written as (1.16). Suppose that $\Phi_{\lambda}$ is a solution of the system (2.14), $\mu \in \boldsymbol{R} \backslash\{0\}$, and $L$ is a real constant $s \times 2(n+1)$ matrix satisfying (3.7). Let $h=L \tau \Phi_{\sqrt{-1} \mu}$ and $\tilde{\Phi}_{\lambda}=\Phi_{\lambda} D_{\lambda}$ where $D_{\lambda}$ is the Darboux matrix given by (3.4). If $\tilde{a}_{j}(0) \neq 0$, 
$\tilde{a}_{s}(0) \neq 0$ for all $j, s$, then there exist an open neighbourhood $U$ around the origin $0 \in M_{1}^{n_{1}}\left(c_{1}\right) \times M_{2}^{n_{2}}\left(c_{2}\right)$ and a local isometric immersion $\tilde{\varphi}: U \rightarrow M^{2 n-1}(c)$ such that $\tilde{\mathbf{r}}=\mathbf{r}_{c} \circ \tilde{\varphi}$ can be expressed explicitly by

$$
\tilde{\mathbf{r}}=\tilde{Q} D_{1}^{-1}(0)\left(\kappa_{1}^{-1} \tilde{\boldsymbol{\Phi}}_{1}^{(1)}+\kappa_{2}^{-1} \tilde{\boldsymbol{\Phi}}_{1}^{(2)}\right)=\tilde{Q} D_{1}^{-1}(0) \Phi_{1}\left(\kappa_{1}^{-1} D_{1}^{(1)}+\kappa_{2}^{-1} D_{1}^{(2)}\right),
$$

where $\tilde{Q}$ is a constant matrix defined by (2.17) with $\tilde{A}(0), \tilde{b}_{1}(0)$ and $\tilde{b}_{2}(0)$.

Remark. The above process of the Darboux transformation is purely algebraic. Hence, starting from a special solution $\Phi_{\lambda}$ to (2.14) for which the corresponding $\mathbf{r}=Q\left(\kappa_{1}^{-1} \Phi_{1}^{(1)}+\kappa_{2}^{-1} \Phi_{1}^{(2)}\right)$ may be degenerated, we can repeat the processes via the purely algebraic algorithm and obtain a sequence of solutions to (2.14): $\Phi_{\lambda} \rightarrow \tilde{\Phi}_{\lambda} \rightarrow \tilde{\tilde{\Phi}}_{\lambda} \rightarrow \cdots$, from which we obtain a sequence of local isometric immersions from $M_{1}^{n_{1}}\left(c_{1}\right) \times M_{2}^{n_{2}}\left(c_{2}\right)$ to $M^{2 n-1}(c)$.

\section{§4. The construction of local isometric immersions from a trivial solution}

For $c \neq 0$ we may take the following trivial solution of (2.11):

$$
F_{r}=0, \quad A=\left(\begin{array}{cccc}
I_{n_{1}-1} & 0 & 0 & 0 \\
0 & \hat{\varepsilon} & 0 & \frac{1}{\sqrt{2}} \\
0 & 0 & I_{n_{2}-1} & 0 \\
0 & \frac{-1}{\sqrt{2}} & 0 & \varepsilon \hat{\varepsilon}
\end{array}\right), \quad b=A E_{n}, \quad \hat{\varepsilon}=\sqrt{1-\frac{\varepsilon}{2}} .
$$

Set

$$
k_{1}=\frac{\kappa_{1}}{v_{1} \sqrt{2}}, \quad k_{2}=\frac{\kappa_{2} \varepsilon \hat{\varepsilon}}{v_{2}} .
$$

For $c=c_{1}=0$, we may take the following trivial solution of (2.11):

$$
F_{r}=0, \quad A=I_{n}, \quad b=A E_{n},
$$

and set

$$
k_{1}=1, \quad k_{2}=v_{2}^{-1} \kappa_{2} .
$$

Thus, by writting $b_{r}^{T}=\left(0, \ldots, k_{r}\right)$, the solution to (2.14) can be expressed as a block matrix

$$
\Phi_{\lambda}=\left(\Phi_{\lambda}^{(i j)}\right), \quad \text { with } \Phi_{\lambda}^{(i j)}=0 \text { for } i+j=\text { odd }, \quad i, j=1, \ldots, 6,
$$

where

$$
\begin{aligned}
& \Phi_{\lambda}^{(11)}=\frac{1}{\chi_{1}^{2}}\left(\varepsilon_{1} k_{1}^{2} X_{n_{1}}+\lambda^{2}\right), \quad \Phi_{\lambda}^{(22)}=\frac{1}{\chi_{2}^{2}}\left(\varepsilon_{2} k_{2}^{2} X_{n}+\varepsilon \lambda^{2}\right), \\
& \Phi_{\lambda}^{(13)}=-\varepsilon_{1}\left(\Phi_{\lambda}^{(31)}\right)^{T}=-\varepsilon_{1}\left(0, \ldots, 0, \frac{k_{1}}{\chi_{1}} Y_{n_{1}}\right),
\end{aligned}
$$




$$
\begin{aligned}
& \Phi_{\lambda}^{(24)}=-\varepsilon_{2}\left(\Phi_{\lambda}^{(42)}\right)^{T}=-\varepsilon_{2}\left(0, \ldots, 0, \frac{k_{2}}{\chi_{2}} Y_{n}\right), \\
& \Phi_{\lambda}^{(15)}=\varepsilon_{1}\left(\Phi_{\lambda}^{(15)}\right)^{T}=\varepsilon_{1}\left(0, \ldots, 0, \frac{\lambda k_{1}}{\chi_{1}^{2}}\left(X_{n_{1}}-1\right)\right), \\
& \Phi_{\lambda}^{(26)}=\frac{\varepsilon_{2}}{\varepsilon}\left(\Phi_{\lambda}^{(62)}\right)^{T}=\varepsilon_{2}\left(0, \ldots, 0, \frac{\lambda k_{2}}{\chi_{2}^{2}}\left(X_{n}-1\right)\right), \\
& \Phi_{\lambda}^{(33)}=\operatorname{diag}\left(X_{1}, \ldots, X_{n_{1}}\right), \quad \Phi_{\lambda}^{(44)}=\operatorname{diag}\left(X_{n_{1}+1}, \ldots, X_{n}\right), \\
& \Phi_{\lambda}^{(35)}=-\Phi_{\lambda}^{(53)}=\operatorname{diag}\left(Y_{1}, \ldots, Y_{n_{1}-1}, \frac{\lambda}{\chi_{1}} Y_{n_{1}}\right), \\
& \Phi_{\lambda}^{(64)}=-J_{\varepsilon} \Phi_{\lambda}^{(46)}=-\operatorname{diag}\left(Y_{n_{1}+1}, \ldots, Y_{n-1}, \frac{\varepsilon \lambda}{\chi_{2}} Y_{n}\right), \\
& \Phi_{\lambda}^{(55)}=\operatorname{diag}\left(X_{1}, \ldots, X_{n_{1}-1}, \frac{1}{\chi_{1}^{2}}\left(\lambda^{2} X_{n_{1}}+\varepsilon_{1} k_{1}^{2}\right)\right), \\
& \Phi_{\lambda}^{(66)}=\operatorname{diag}\left(X_{n_{1}+1}, \ldots, X_{n-1}, \frac{1}{\chi_{2}^{2}}\left(\varepsilon \lambda^{2} X_{n}+\varepsilon_{2} k_{2}^{2}\right)\right), \\
& \chi_{1}=\chi_{1}(\lambda)=\sqrt{\lambda^{2}+\varepsilon_{1} k_{1}^{2}}, \quad \chi_{2}=\chi_{2}(\lambda)=\sqrt{\varepsilon \lambda^{2}+\varepsilon_{2} k_{2}^{2}}, \\
& X_{I}=\cos \left(\lambda x_{I}\right)\left(I \neq n_{1}, n\right), \quad X_{n_{1}}=\cos \left(\chi_{1} x_{n_{1}}\right), \quad X_{n}=\cos \left(\chi_{2} x_{n}\right), \\
& Y_{I}=\sin \left(\lambda x_{I}\right)\left(I \neq n_{1}, n\right), \quad Y_{n_{1}}=\sin \left(\chi_{1} x_{n_{1}}\right), \quad Y_{n}=\sin \left(\chi_{2} x_{n}\right) .
\end{aligned}
$$

For $\mu \in \boldsymbol{R}$, we choose the following constant $2 \times 2(n+1)$ matrix $L$ :

$$
\begin{aligned}
L & =\left(\begin{array}{cccccc}
L_{1} & 0 & L_{3} & 0 & L_{5} & 0 \\
0 & L_{2} & 0 & L_{4} & 0 & L_{6}
\end{array}\right), \\
& =\left(\begin{array}{cccccc}
l_{0} & 0 & l_{1} \cdots l_{n_{1}} & 0 & l_{n+1} \cdots l_{n+n_{1}} & 0 \\
0 & l_{0}^{\prime} & 0 & l_{n_{1}+1} \cdots l_{n} & 0 & l_{n+n_{1}+1} \cdots l_{2 n}
\end{array}\right),
\end{aligned}
$$

such that

$$
\begin{gathered}
\sum_{j} l_{n+j}^{2}=\sum_{j} l_{j}^{2}+\varepsilon_{1} l_{0}^{2} \neq 0, \quad \sum_{s \neq n} l_{n+s}^{2}+\varepsilon l_{2 n}^{2}=\sum_{s} l_{s}^{2}+\varepsilon_{2} l_{0}^{2} \neq 0, \\
k_{1} l_{n+n_{1}}-\mu l_{0}=0, \quad k_{2} l_{2 n}-\mu l_{0}^{\prime}=0 .
\end{gathered}
$$

It is easy to see that $L$ defined by (4.4) satisfies (3.3) and (3.7). Thus, we have

$$
\begin{aligned}
h & =L \tau \Phi_{\sqrt{-1} \mu}=\left(\begin{array}{cccccc}
h_{1} & 0 & h_{3} & 0 & \sqrt{-1} h_{5} & 0 \\
0 & h_{2} & 0 & h_{4} & 0 & \sqrt{-1} h_{6}
\end{array}\right) \\
& =\left(\begin{array}{ccccccc}
\xi_{1} & 0 & \eta_{1} \cdots \eta_{n_{1}} & 0 & \sqrt{-1} \zeta_{1} \cdots \sqrt{-1} \zeta_{n_{1}} & 0 \\
0 & \xi_{2} & 0 & \eta_{n_{1}+1} \cdots \eta_{n} & 0 & \sqrt{-1} \zeta_{n_{1}+1} \cdots \sqrt{-1} \zeta_{n}
\end{array}\right),
\end{aligned}
$$


where

$$
\begin{aligned}
& \xi_{1}=l_{0} \cos \left(\gamma_{1} \sqrt{-1} x_{n_{1}}\right)-l_{n_{1}} \sqrt{-1} \gamma_{1}^{-1} k_{1} \sin \left(\gamma_{1} \sqrt{-1} x_{n_{1}}\right)=\mu^{-1} k_{1} \zeta_{n_{1}}, \\
& \xi_{2}=l_{0}^{\prime} \cos \left(\gamma_{2} \sqrt{-1} x_{n}\right)-l_{n} \sqrt{-1} \gamma_{2}^{-1} k_{2} \sin \left(\gamma_{2} \sqrt{-1} x_{n}\right)=\mu^{-1} k_{2} \zeta_{n}, \\
& \eta_{I}=l_{I} \operatorname{ch}\left(\mu x_{I}\right)+l_{n+I} \operatorname{sh}\left(\mu x_{I}\right), \quad\left(I \neq n_{1}, n\right) \\
& \eta_{n_{1}}=l_{n_{1}} \cos \left(\gamma_{1} \sqrt{-1} x_{n_{1}}\right)-l_{0} \sqrt{-1} k_{1}^{-1} \gamma_{1} \sin \left(\gamma_{1} \sqrt{-1} x_{n_{1}}\right), \\
& \eta_{n}=l_{n} \cos \left(\gamma_{2} \sqrt{-1} x_{n}\right)-l_{0}^{\prime} \sqrt{-1} k_{2}^{-1} \gamma_{2} \sin \left(\gamma_{2} \sqrt{-1} x_{n}\right), \\
& \zeta_{I}=l_{I} \operatorname{sh}\left(\mu x_{I}\right)+l_{n+I} \operatorname{ch}\left(\mu x_{I}\right), \quad\left(I \neq n_{1}, n\right), \\
& \gamma_{r}=-\sqrt{-1} \chi_{r}(\sqrt{-1} \mu) .
\end{aligned}
$$

It is clear that

$$
h_{i}(0)=L_{i} \quad \text { for } i=1, \ldots, 6 .
$$

When $\gamma_{r}=0$, we have

$$
\xi_{1}=l_{0}, \quad \xi_{2}=l_{0}^{\prime}, \quad \zeta_{n}=l_{2 n}, \quad \zeta_{n_{1}}=l_{n+n_{1}} .
$$

It is easy to see that $\rho=-h^{\prime} \sigma J h^{*}$ is a $2 \times 2$ diagonal matrix. Since $\rho$ is skewsymmetric, then we get $\rho=0$. Thus, by (3.9) (3.11), we have

$$
\begin{aligned}
& \Delta=\left(\begin{array}{cc}
\Delta_{1} & 0 \\
0 & \Delta_{2}
\end{array}\right), \quad \Delta_{1}=h_{5} h_{5}^{T}=\sum_{i} \zeta_{i}^{2}, \quad \Delta_{2}=h_{6} h_{6}^{T} \sum_{s} \zeta_{s}^{2}, \\
& D_{1}^{(1)}=\frac{1}{\Delta_{1}\left(1+\mu^{2}\right)}\left(\left(1+\mu^{2}\right) \Delta_{1}-2 \varepsilon_{1} k_{1}^{2} \zeta_{n_{1}}^{2}, 0,-2 \mu k_{1} \zeta_{n_{1}} h_{3}, 0,-2 k_{1} \zeta_{n_{1}} h_{5}, 0\right)^{T}, \\
& D_{1}^{(2)}=\frac{1}{\Delta_{2}\left(1+\mu^{2}\right)}\left(0,\left(1+\mu^{2}\right) \Delta_{2}-2 \varepsilon_{2} k_{2}^{2} \zeta_{n}^{2}, 0,-2 \mu k_{2} \zeta_{n} h_{4}, 0,-2 k_{2} \zeta_{n} h_{6}\right)^{T}, \\
& \tilde{b}_{1}=\frac{k_{1}}{\Delta_{1}}\left(-2 \zeta_{n_{1}} \hat{h}_{5}, \hat{h}_{5} \hat{h}_{5}^{T}-\zeta_{n_{1}}^{2}\right)^{T}, \quad \tilde{b}_{2}=\frac{k_{2}}{\Delta_{2}}\left(-2 \zeta_{n} \hat{h}_{6}, \hat{h}_{6} \hat{h}_{6}^{T}-\zeta_{n}^{2}\right)^{T},
\end{aligned}
$$

and

$$
\tilde{A}=\left(\begin{array}{cccc}
I_{n_{1}-1}-\frac{2}{\Delta_{1}} \hat{h}_{5}^{T} \hat{h}_{5} & -\frac{2 \hat{\varepsilon}}{\Delta_{1}} \zeta_{n_{1}} \hat{h}_{5}^{T} & 0 & -\frac{\sqrt{2}}{\Delta_{1}} \zeta_{n_{1}} \hat{h}_{5}^{T} \\
-\frac{2}{\Delta_{1}} \zeta_{n_{1}} \hat{h}_{5} & \hat{\varepsilon}\left(1-\frac{2}{\Delta_{1}} \zeta_{n_{1}}^{2}\right) & 0 & \frac{\sqrt{2}}{2}-\frac{\sqrt{2}}{\Delta_{1}} \zeta_{n_{1}}^{2} \\
0 & \frac{\sqrt{2}}{\Delta_{2}} \zeta_{n} \hat{h}_{6}^{T} & I_{n_{2}-1}-\frac{2}{\Delta_{2}} \hat{h}_{6}^{T} \hat{h}_{6} & -\frac{2 \varepsilon \hat{\varepsilon}}{\Delta_{2}} \zeta_{n} \hat{h}_{6}^{T} \\
0 & \frac{\sqrt{2}}{\Delta_{2}} \zeta_{n}^{2}-\frac{\sqrt{2}}{2} & -\frac{2}{\Delta_{2}} \zeta_{n} \hat{h}_{6} & \varepsilon \hat{\varepsilon}\left(1-\frac{2}{\Delta_{2}} \zeta_{n}^{2}\right)
\end{array}\right) \quad(c \neq 0),
$$




$$
\tilde{A}=\left(\begin{array}{cc}
I_{n_{1}}-\frac{2}{\Delta_{1}} h_{5}^{T} h_{5} & 0 \\
0 & I_{n_{2}}-\frac{2}{\Delta_{2}} h_{6}^{T} h_{6}
\end{array}\right) \quad(c=0),
$$

where

$$
\hat{h}_{1}=\left(\zeta_{1}, \ldots, \zeta_{n_{1}-1}\right), \quad \hat{h}_{2}=\left(\zeta_{n_{1}+1}, \ldots, \zeta_{n-1}\right) .
$$

By Theorem 3.4, if $\tilde{a}_{j}(0) \neq 0, \tilde{a}_{s}(0) \neq 0$ for all $j, s$, then there exist an open neighbourhood $U$ around the point $x=0$ in $M_{1}^{n_{1}}\left(c_{1}\right) \times M_{2}^{n_{2}}\left(c_{2}\right)$ and a nondegenerated locally isometric immersion $\tilde{\varphi}: U \rightarrow M^{2 n-1}(c)$, such that its position vector in $\boldsymbol{R}^{2 n}$ is

$$
\tilde{\mathbf{r}}=\mathbf{r}_{c} \circ \tilde{\varphi}=\tilde{Q} D_{1}^{-1}(0)\left(\kappa_{1}^{-1} \tilde{\Phi}_{1}^{(1)}+\kappa_{2}^{-1} \tilde{\Phi}_{1}^{(2)}\right)=\tilde{Q} D_{1}^{-1}(0) \Phi_{1}\left(\kappa_{1}^{-1} D_{1}^{(1)}+\kappa_{2}^{-1} D_{1}^{(2)}\right),
$$

where $\tilde{Q}$ is a constant matrix defined by $(2.17)$ with $\tilde{A}(0), \tilde{b}_{1}(0)$ and $\tilde{b}_{2}(0)$. Hence, it is sufficient to choose suitably $L$ such that

$$
l_{n+I} \neq 0, \quad \sum_{i \neq n_{1}} l_{n+i}^{2}-l_{n+n_{1}}^{2} \neq 0, \quad \sum_{s \neq n} l_{n+s}^{2}-l_{2 n}^{2} \neq 0 .
$$

Thus, starting from a trivial solution $\Phi_{\lambda}$ and using the Darboux transformation $\tilde{\Phi}_{\lambda}=\Phi_{\lambda} D_{\lambda}$ and (3.12), we can construct a series of locally isometric immersions from $M_{1}^{n_{1}}\left(c_{1}\right) \times M_{2}^{n_{2}}\left(c_{2}\right)$ to $M^{2 n-1}(c)$.

Remark. It should be remarked that we may choose constant matrices $L$ satisfying (4.5) and (4.9) in a quite arbitrary way. For example, according to the signs of $\gamma_{1}^{2}=\mu^{2}-\varepsilon_{1} k_{1}^{2}$ and $\gamma_{2}^{2}=\varepsilon \mu^{2}-\varepsilon_{2} k_{2}^{2}$, we can choose $L$ as follows. If $\gamma_{1}^{2} \geq 0$ and $\gamma_{2}^{2}<0$, then $L$ may be taken as

$$
L=\left(\begin{array}{cccccc}
\mu^{-1} k_{1} l_{n_{1}} & 0 & L_{3} & 0 & L_{5} & 0 \\
0 & \mu^{-1} k_{2} l_{n-1} & 0 & L_{4} & 0 & L_{6}
\end{array}\right),
$$

where

$$
\begin{gathered}
L_{3}=\left(l_{1}, \ldots, l_{n_{1}-1}, \mu^{-1} \gamma_{1} l_{n_{1}}\right), \quad L_{5}=\left(l_{1}, \ldots, l_{n_{1}}\right), \\
L_{4}=\left(l_{n_{1}+1}, \ldots, l_{n-2}, 0,0\right), \quad L_{6}=\left(l_{n_{1}+1}, \ldots, l_{n-2}, \sqrt{-1} \mu^{-1} \gamma_{2} l_{n-1}, l_{n-1}\right),
\end{gathered}
$$

such that

$$
l_{I} \neq 0, \quad \sum_{i \neq n_{1}} l_{i}^{2}-l_{n_{1}}^{2} \neq 0, \quad \sum_{s \neq n-1, n} l_{s}^{2}+\left(\varepsilon_{2} \mu^{-2} k_{2}^{2}-\varepsilon-1\right) l_{n-1}^{2} \neq 0 .
$$

\section{REFERENCES}

[CK] S.-s. CHERN AND N. H. KuIPER, Some theorems on the isometric imbedding of compact Riemann manifolds in Euclidean space, Ann. of Math. (2), 56 (1952), 422-430.

[FP] D. Ferus AND F. PEDIT, Isometric immersions of space forms and soliton theory, Math. Ann., 305 (1996), 329-342. 
[Hi] D. Hilbert, Über Flächen von konstanter Gausscher Krümmung, Trans. Amer. Math. Soc., 2 (1901), 87-99.

[HS] Q. He AND Y. B. SHEN, Explicit construction for local isometric immersions of space forms, to appear in Chinese Ann. Math. Ser. B.

[Mo] J. D. Moore, Isometric immersions of space forms in space forms, Pacific J. Math., 40 (1972), 157-166.

[Pe] F. J. Pedit, A non-immersion theorem for space forms, Comment. Math. Helv., 63 (1988), 672-674.

[Ter] C.-L. Terng, Soliton equations and differential geometry, J. Differential Geom., 45 (1997), 407-445.

[TU] C.-L. Terng And K. Uhlenbeck, Bäcklund transformation and loop group actions, Comm. Pure Appl. Math., 53 (2000), 1-75.

[Xa] F. XAVIER, A non-immersion theorem for hyperbolic manifolds, Comment. Math. Helv., 60 (1985), 280-283.

[Zh] Z. X. ZHou, Darboux transformations for the twisted so $(p, q)$ system and local isometric immersion of space forms, Inverse Problems, 14 (1998), 1353-1370.

Department of Applied Mathematics

TONGJI UNIVERSITY

Shanghai 200092, China

e-mail: Qun_he@163.com

Department of Mathematics

West Brook Campus

ZHEJIANG UNIVERSITY

Hangzhou 310028, China

e-mail: ybsh@mail.hz.zj.cn 